\title{
Psicologia e Odontopediatria: A Contribuição da Análise Funcional do Comportamento
}

\author{
Antonio Bento Alves de Moraes' \\ Universidade de Campinas \\ Kira Anayansi Singh Sanchez. \\ Universidade Estadual Paulista \\ Rosana de Fátima Possobon \\ Universidade de Campinas \\ Áderson Luiz Costa Júnior \\ Universidade de Brasília
}

\begin{abstract}
Resumo
Objetivando analisar funcionalmente a atuação do odontopediatra, procedeu-se ao atendimento odontológico de 3 crianças nãocolaboradoras, utilizando ansiolítico ou placebo. As sessões foram filmadas e registrou-se os eventos clínicos e comportamentais dos participantes, em intervalos de 15 segundos. Os resultados revelaram que a colaboração das crianças pode ser considerada condição estabelecedora para os comportamentos da profissional. O ansiolítico não demonstrou efeitos sobre o comportamento dos pacientes. "Direção", categoria comportamental predominantemente utilizada pela dentista, revelou-se eficaz na evocação de respostas de colaboração para 2 pacientes. Os dados expressam a contribuição da análise funcional do comportamento ao estudo da interação profissional-paciente em odontopediatria.

Palavras-chave: Relações dentista-paciente; psicologia aplicada à odontologia; repertório comportamental; análise funcional.
\end{abstract}

Psychology and Pediatric Dentistry: The Contribution of Behaviour Functional Analysis

\begin{abstract}
The aim of this study was to carry out a functional description of the pediatric dentists' behavior concerning professional performance. Subjects were 3 uncooperative dental patients who were treated using placebo or diazepam. Dental sessions were videotaped. Procedures and behaviors were recorded in 15-second intervals. Results revealed that children's level of cooperative behavior may be a considered variable that affect professional behaviors. Diazepam was not effective. "Guidance" was predominantly used by the dentist and evoked cooperative behavior with two patients. The results express the contribution of behaviour functional analysis to the study of patterns of interaction between the dental practitioner and child patient.

Keywords: Dentist-patient relationship; psychology applied to dentistry; dentist's behavior; functional analysis.
\end{abstract}

Alguns estudos sugerem que o repertório de comportamentos da criança, exposta à situação de atendimento odontológico, resulta do manejo inadequado do cirurgião-dentista (Prins, Weerkamp, Horst, Jong \& Tan, 1987; Weinstein, Getz, Ratener \& Domoto, 1982). Entretanto, poucos autores analisam, especificamente, o comportamento do profissional e como este é afetado funcionalmente pelo comportamento do paciente. A habilidade para interagir e se comunicar com o paciente é essencial para criar uma boa relação profissional. Caldana e Biasoli-Alves (1990) ressaltam que conhecer o comportamento do paciente permite um melhor desempenho odontológico. Ten-Berge, Veerkamp e Hoogstraten (1999) observam que o comportamento do dentista exerce influência relevante sobre o desenvolvimento do medo de tratamento odontológico em crianças.

De maneira geral, os odontopediatras reconhecem a necessidade de melhorar e expandir seus conhecimentos sobre manejo do comportamento de crianças, mas a exposição à programas de treinamento e a aceitação de novas estratégias,

\footnotetext{
${ }^{1}$ Endereço para correspondência: Faculdade de Odontologia de Piracicaba, UNICAMP, Av. Limeira, 901, Areião, Caixa Postal 52, 14414 903, Piracicaba, SP. Fone: (19) 34125276, Fax: (19) 34125218. E-mail: abento@fop.unicamp.br
}

baseadas em princípios psicológicos, têm sido extremamente lentas (Allen, Stanley \& McPherson, 1990). Conforme descreve Costa Jr. (2001), poucos trabalhos em psicologia têm estudado sistematicamente o efeito de intervenções psicossociais sobre o comportamento de crianças expostas a consultas e tratamentos de saúde, principalmente quando as contingências envolvem procedimentos médicos e/ou odontológicos invasivos.

Trabalhos recentes, descritos por Kazdin (1993) e por Costa Jr. (2001), sugerem que, uma proposta para estudar o comportamento dos profissionais de saúde, no contexto do atendimento clínico, deveria incluir a análise funcional do comportamento de todos os indivíduos envolvidos na situação. A análise funcional se refere à investigação das relações entre as respostas de um indivíduo aos estímulos ambientais objetivamente identificados (Souza, 1997). Conforme aponta Meyer (1997), uma análise funcional é essencial em estudos cujo objetivo inclua a predição e/ou o controle de repertórios de comportamento em situações específicas.

O presente trabalho insere-se na interface psicologiaodontologia e destaca a importância de estudos 
comportamentais que ampliem a visibilidade dos aspectos psicológicos presentes na interação dentista-criança, considerando-se as características potencialmente aversivas da situação de tratamento odontológico e as típicas manifestações comportamentais do dentista e da criança. Neste contexto, a análise funcional do comportamento revela-se como uma abordagem adequada ao estudo do comportamento presente nesta situação.

O objetivo deste estudo foi descrever e analisar o comportamento do odontopediatra, bem como as variáveis clínicas e comportamentais que o afetam, utilizando as contribuições da análise funcional do comportamento.

\section{Método}

O projeto do presente trabalho foi avaliado e aprovado pelo Comitê de Ética em Pesquisa da Faculdade de Odontologia de Piracicaba, da Universidade Estadual de Campinas. Todos os participantes estavam devidamente informados sobre a natureza da pesquisa e o seu nível de envolvimento.

\section{Participantes}

Participaram do estudo uma observadora treinada, uma dentista com 10 anos de experiência em atendimento de crianças e 3 crianças do sexo masculino (P1, P2 e P3), com idade entre 4 e 5 anos e história anterior de não-colaboração, manifestada com outros profissionais de Odontologia. Para P1 e P2 foram realizadas cinco sessões e para P3, nove sessões de atendimento odontológico. As sessões foram realizadas com um intervalo de 7 dias.

\section{Procedimentos}

A dentista seguiu a mesma seqüência de procedimentos clínicos para todos os pacientes. Na primeira sessão, designada de "linha de base", a criança foi instruída sobre os procedimentos que seriam realizados e como ela deveria se comportar. Em seguida, a dentista mostrou alguns brinquedos que seriam usados ao final da sessão, caso a criança colaborasse, e logo depois realizou treino de escovação, profilaxia e aplicação tópica de flúor. As sessões de atendimento subseqüentes incluíam duas sessões com o uso de um ansiolítico (diazepam) por via oral, na dosagem de $0,3 \mathrm{mg} / \mathrm{Kg}$ de peso, administrado 60 minutos antes do início da sessão, ou duas sessões com placebo também via oral, em igual volume e 60 minutos antes do início do atendimento, e assim, alternadamente, até o final do tratamento. Na literatura, não há unanimidade em relação à dose de diazepam recomendada. Trapp (1981) considera a dose adequada para crianças jovens entre 0,2 e $0,5 \mathrm{mg} / \mathrm{Kg}$ de peso. A Federation Dentaire Internationale (1989) recomenda a dose de $0,2 \mathrm{mg} / \mathrm{Kg}$ de peso 1 hora antes da intervenção e Andrade (1999) recomenda doses entre 0,1 e 0,3 mg/Kg.
Em Odontologia, as drogas ansiolíticas têm sido utilizadas como pré-medicação em pacientes temerosos ou apreensivos (Fuchs \& Wannamacher, 1992). Corretamente utilizado para sedação consciente, o benzodiazepinico pode aumentar o bem estar do paciente durante o tratamento odontológico e, com isso, melhorar sua qualidade (Mindus, 1987). A droga por si só não reduz a não - colaboração, mas pode diminuir a ansiedade e, como decorrência, atuar como agente facilitador da interação profissional - paciente (Possobon, 2000).

O delineamento utilizado foi do tipo duplo-cego, isto é, a dentista, o paciente e sua mãe não sabiam quando era oferecida a droga ou o placebo.

A dentista foi orientada a realizar o tratamento respeitando a seguinte regra: "não utilizar restrição física durante as primeiras cinco sessões de atendimento". Atividades recreativas, tais como contar estórias e brincar com fantoches, denominadas de atividade lúdica, foram realizadas ao final de todas as sessões em que a criança colaborou.

Todas as sessões foram gravadas em videoteipe, com sons de "bip" a cada 15 segundos. Após as filmagens, a observadora assistiu as fitas, registrando os eventos clínicos e comportamentais ocorridos em cada intervalo de 15 segundos. Baseando-se nos registros observacionais, foram identificadas categorias de comportamento da dentista e da criança. $\mathrm{Na}$ análise dos dados procurou-se investigar as relações entre as ações da dentista e variáveis presentes na situação odontológica. Para isto, foram identificadas as respostas do paciente, a atuação da dentista frente a cada resposta e a conseqüência desta atuação em diferentes momentos do tratamento.

Além disso, os procedimentos clínicos também foram considerados como variáveis que podiam influenciar os comportamentos do paciente ou da dentista. A Tabela 1 apresenta as categorias de comportamento da dentista e da criança e suas respectivas definições.

\section{Resultados}

A Tabela 2 apresenta a duração das sessões de atendimento e as rotinas odontológicas realizadas com os pacientes P1, P2 e P3.

Ao final de todas as sessões de P1 e P2, foi realizada atividade lúdica. Com P3, a atividade lúdica ocorreu apenas a partir da sexta sessão, pois a criança não colaborou nas sessões anteriores. A partir da segunda sessão de P1 e P2, foi realizado o tratamento curativo que incluiu anestesia, exodontia e dentística. Para P3, não foi possível realizar qualquer tarefa clínica da segunda até a quinta sessão, sendo realizada anestesia e dentística a partir da sexta sessão.

A Tabela 3 apresenta o percentual de ocorrência das categorias de comportamento da dentista e dos pacientes. Os comportamentos da dentista podem ser agrupados em três categorias funcionais: a) realiza atividade (que se refere 
Tabela 1

Categorias de Comportamento da Dentista e dos Pacientes e suas Respectivas Definições

\begin{tabular}{|c|c|}
\hline Categorias da dentista & Definição \\
\hline Realiza atividade & Atividades técnicas que envolvem a execução dos procedimentos odontológicos \\
\hline Direção & $\begin{array}{l}\text { Ordens, instruções, orientações, estabelecimento de regras, explicações sobre o procedimento } \\
\text { e o comportamento desejado. }\end{array}$ \\
\hline Tranqüilização & Perguntas sobre sentimentos e sensações; agrado físico. \\
\hline Distração & $\begin{array}{l}\text { Eventos como "contar estórias", "cantar" ou colocar fitas com músicas com o intuito de } \\
\text { desviar a atenção do paciente e competir com outros estímulos, presentes na situação } \\
\text { odontológica. }\end{array}$ \\
\hline Relaxamento & $\begin{array}{l}\text { Ensinar a criança, por meio de instrução verbal ou modelos, a realizar exercícios de respiração; } \\
\text { massagens faciais ou nos braços do paciente; solicitação de relaxamento. }\end{array}$ \\
\hline Persuasão & $\begin{array}{l}\text { Verbalizações para convencer a criança a colaborar oferecendo algum tipo de recompensa } \\
\text { (brinde, brincar após a sessão) ou usar frases do tipo: "me ajude" ou "a mamãe está ficando } \\
\text { triste". }\end{array}$ \\
\hline Punição verbal & Repreensão verbal, ameaças de não brincar se não colaborar. \\
\hline Restrição física & $\begin{array}{l}\text { Contenção dos movimentos da criança pela mãe, auxiliar ou dentista para realizar o tratamento } \\
\text { e impedir ferimentos, principalmente durante a rotina de anestesia e preparo cavitário. }\end{array}$ \\
\hline Categorias dos pacientes & Definição \\
\hline Colaboração & Permite a realização do tratamento, segue instruções e ordens. \\
\hline Não-colaboração & $\begin{array}{l}\text { Qualquer comportamento da criança que interrompe a atuação do dentista durante o tratamento } \\
\text { odontológico. }\end{array}$ \\
\hline Colaboração sob restrição & Criança permite a realização do tratamento odontológico, sob contenção física. \\
\hline
\end{tabular}

à execução das rotinas odontológicas, conforme a Tabela 1); b) estratégias positivas (que incluem as intervenções de manejo comportamental do paciente, tais como direção, tranqüilização, relaxamento e persuasão); e c) estratégias negativas (usualmente consideradas desagradáveis ou aversivas e que envolvem restrição física e punição verbal).

As categorias de comportamento dos pacientes foram classificadas funcionalmente em dois grupos: a) comportamentos concorrentes que se referem às respostas da criança que impedem a atuação do dentista (movimentos da cabeça e/ou do corpo e choro), isto é, respostas de nãocolaboração; e b) comportamentos não concorrentes, os quais incluem respostas da criança que não dificultam e/ou tendem a facilitar a atuação do dentista.

Para obtenção dos percentuais de ocorrência de cada categoria de comportamento, considerou-se a duração total, em minutos, de todas as sessões realizadas com cada paciente, sendo cada minuto dividido em 4 intervalos de 15 segundos. No intuito de registrar todos os comportamentos do dentista que ocorriam a cada intervalo, foi necessário incluir categorias diferentes em um mesmo intervalo, ou seja, a dentista poderia estar "realizando atividade" e "distraindo" a criança ao mesmo tempo. Portanto, as categorias não foram excludentes.

Para P1 $(N=456)$, a categoria de comportamento da dentista "realiza atividade" ocorreu em $67,4 \%$ do tempo de atendimento e as "estratégias positivas" (direção, tranqülização e distração) ocorreram em $66,4 \%$. Em relação ao paciente, os "comportamentos não concorrentes" ocorreram em $92,7 \%$ do tratamento e os "comportamentos concorrentes" em 7,3\% do tratamento.

Para P2 ( $N=504)$, a categoria "realiza atividade" ocorreu em $85,9 \%$ do tempo de tratamento. As "estratégias positivas" ocorreram em $87,2 \%$ do tempo e as "estratégias negativas" (punição verbal) em apenas $0,79 \%$. Os "comportamentos não concorrentes" ocorreram em $93,8 \%$ do tempo e os "comportamentos concorrentes" em 6,2\% do tratamento.

Durante as primeiras 5 sessões de atendimento de P3, quando não houve restrição física, o número de intervalos foi 404. A categoria "realiza atividade" ocorreu em apenas $8,1 \%$ do tempo de tratamento. As "estratégias positivas" ocorreram em 78,5\% e as "estratégias negativas" (punição verbal) em 9,0\% do tempo. Os "comportamentos não concorrentes" de P3 ocorreram em 28,3\% e os "comportamentos concorrentes" aconteceram em 71,7\% dos 404 intervalos. Para o mesmo paciente, da sexta até a nona sessão, quando a restrição física foi introduzida, o número total de intervalos foi de 432. A categoria de comportamento da dentista "realiza atividade" ocorreu em 62,0\% do tempo de tratamento. As "estratégias positivas" ocorreram em 50,1\% do tempo e as "estratégias negativas" em 37,4\% do atendimento. Os "comportamentos concorrentes" de P3 ocorreram em 17,7\% do tempo e os "comportamentos não concorrentes" ocorreram em $82,3 \%$ do tempo, sendo $42,8 \%$ sem restrição física e 39,5\% com restrição física. 
Tabela 2

Pacientes, Número de Sessões, Etapa do Trabalho e Procedimento Realizados

\begin{tabular}{|c|c|c|c|c|c|}
\hline & \multicolumn{5}{|c|}{ Sessões do Paciente 1} \\
\hline \multirow{7}{*}{$\begin{array}{l}\text { Etapa } \\
\text { Rotinas }\end{array}$} & 1 (26 min.) & 2 (24min.) & 3 (31 min.) & 4 (14 min.) & 5 (19 min.) \\
\hline & $\mathrm{LB}$ & Placebo & Placebo & Diazepam & Diazepam \\
\hline & Entrada & Entrada & Entrada & Entrada & Entrada \\
\hline & $\mathrm{TE}$ & Anestesia & Anestesia & Anestesia & Anestesia \\
\hline & Profilaxia & Dentística & Dentística & Exodontia & Exodontia \\
\hline & ATF & AL (6 min.) & $\operatorname{AL}(5 \mathrm{~min})$. & AL (6min.) & AL (4min.) \\
\hline & $\mathrm{AL}(4 \mathrm{~min})$. & & & & \\
\hline \multicolumn{6}{|c|}{ Sessões do Paciente 2} \\
\hline \multirow{8}{*}{$\begin{array}{l}\text { Etapa } \\
\text { Rotinas }\end{array}$} & 1 (26 min.) & 2 (25min.) & 3 (20 min.) & 4 (27 min.) & \\
\hline & $\mathrm{LB}$ & Placebo & Placebo & Diazepam & 5 (28 min.) \\
\hline & Entrada & Entrada & Entrada & Entrada & Diazepam \\
\hline & $\mathrm{TE}$ & Anestesia & Anestesia & Anestesia & Entrada \\
\hline & Profilaxia & Dentística & Dentística & Exodontia & Anestesia \\
\hline & ATF & AL (7 min.) & AL (4 min.) & AL (6 min.) & Exodontia \\
\hline & AL (4 min.) & & & & \\
\hline & \multicolumn{5}{|c|}{ Sessões do Paciente 3 (sem restrição física) } \\
\hline \multirow{6}{*}{$\begin{array}{l}\text { Etapa } \\
\text { Rotinas }\end{array}$} & 1 (21 min.) & 2 (20min.) & 3 (26min.) & 4 (16min.) & \\
\hline & Entrada & Diazepam & Diazepam & Placebo & 5 (18min.) \\
\hline & $\mathrm{TE}$ & Entrada & Entrada & Entrada & Placebo \\
\hline & Profilaxia & Insucesso & Insucesso & Insucesso & Entrada \\
\hline & ATF & & & & Insucesso \\
\hline & \multicolumn{5}{|c|}{ Sessões do Paciente 3 (com restrição física) } \\
\hline \multirow{7}{*}{$\begin{array}{l}\text { Etapa } \\
\text { Rotinas }\end{array}$} & $6(22$ min. $)$ & 7 (25min.) & 8 (27 min.) & 9 (34 min.) & \\
\hline & Diazepam & Diazepam & Placebo & Placebo & \\
\hline & Entrada & Entrada & Entrada & Entrada & \\
\hline & $\mathrm{TE}$ & Anestesia & Anestesia & Anestesia & \\
\hline & Profilaxia & Dentística & Dentística & Dentística & \\
\hline & ATF & AL (10 min.) & $\mathrm{AL}(10 \mathrm{~min})$. & AL (8 min.) & \\
\hline & AL (5 min.) & & & & \\
\hline
\end{tabular}

Nota. LB: Linha de Base; TE: Treino de Escovação; ATF: Aplicação Tópica de Flúor; AL: Atividade Lúdica.

A Figura 1 apresenta as freqüências acumuladas de categorias de comportamento da dentista e do paciente P3 durante as cinco primeiras sessões de atendimento. A variabilidade nos padrões de comportamento da dentista e do paciente, observada durante o atendimento de P3 (e não durante o atendimento de P1 e P2), foi o critério utilizado para a apresentação exclusiva desta análise para P3. A ocorrência da categoria "realiza atividade" foi muito baixa na primeira sessão. Observou-se uma discreta ascendência da curva desta categoria na terceira sessão e nenhuma ocorrência na quarta e quinta sessões. Para o comportamento "direção", a curva revelou uma tendência crescente da primeira para a segunda sessão. A curva representativa do comportamento "persuasão" indicou um aumento da freqüência acumulada a partir da terceira sessão. Respostas de "direção" diminuem e respostas de "persuasão" aumentam. Este padrão comportamental sugere que a dentista procura uma conduta que seja mais eficaz em termos de produzir colaboração do paciente.
Considerando-se os comportamento do paciente, a curva referente à "colaboração" teve inicialmente uma tendência ascendente discreta até o final da segunda sessão, observandose um aumento da freqüência acumulada até a quinta sessão.

A Figura 2 se refere às freqüências acumuladas das categorias de comportamento da dentista e do paciente P3 durante as quatro últimas sessões de atendimento odontológico. A curva da categoria "realiza atividade" apresentou uma tendência ascendente da sexta até a nona sessão. Nestas sessões, a dentista conseguiu grande produtividade clínica. A curva do comportamento "direção" revelou valores muito baixos da sexta até a nona sessão e o comportamento "persuasão" praticamente não ocorreu nas últimas quatro sessões de atendimento. Sugere-se que a "restrição física" torna o esforço para persuadir desnecessário. A curva do comportamento "restrição física" mostrou uma tendência crescente na sexta, sétima e metade de oitava sessão.

A categoria "colaboração sob restrição física" ocorreu de forma crescente na sexta e sétima sessões. A partir da metade da oitava sessão e durante a nona sessão, este padrão 
Tabela 3

Percentual de Ocorrência das Categorias de Comportamentos da Dentista e dos Pacientes (P1, P2 e P3) durante o Tratamento Odontológico ( $N=$ numero de intervalos)

\begin{tabular}{llllll}
\hline Participantes & Categorias de comportamento & \multicolumn{3}{c}{ Pacientes } \\
\hline \multirow{3}{*}{ Dentista } & & $\mathrm{P} 1$ & $\mathrm{P} 2$ & $\mathrm{P} 3$ & $\mathrm{P} 3(\mathrm{RF})$ \\
& & $\mathrm{N}=456$ & $\mathrm{~N}=504$ & $\mathrm{~N}=404$ & $\mathrm{~N}=432$ \\
& Realiza atividade & $67,4 \%$ & $85,9 \%$ & $8,16 \%$ & $62,0 \%$ \\
& Estratégias positivas & $66,4 \%$ & $87,2 \%$ & $78,5 \%$ & $50,1 \%$ \\
\multirow{3}{*}{ Paciente } & Estratégias negativas & 0 & $0,79 \%$ & $9,0 \%$ & $37,4 \%$ \\
& & & & & \\
& Comportamentos concorrentes & $7,3 \%$ & $6,2 \%$ & $71,7 \%$ & $17,7 \%$ \\
& Comportamentos não concorrentes & $92,7 \%$ & $93,8 \%$ & $28,3 \%$ & $82,3 \%$ \\
\hline
\end{tabular}

RF: Restrição Física

\section{Dentista}

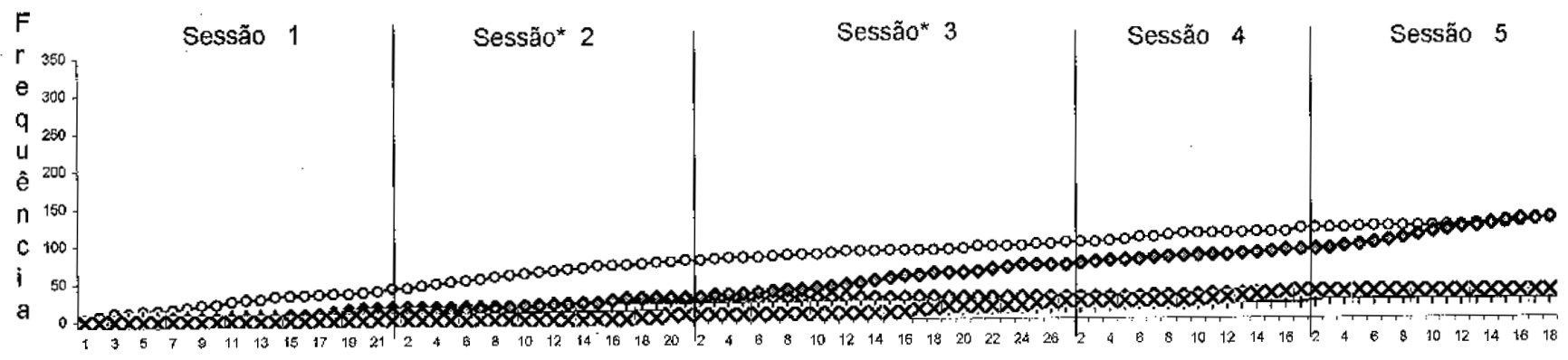

P3

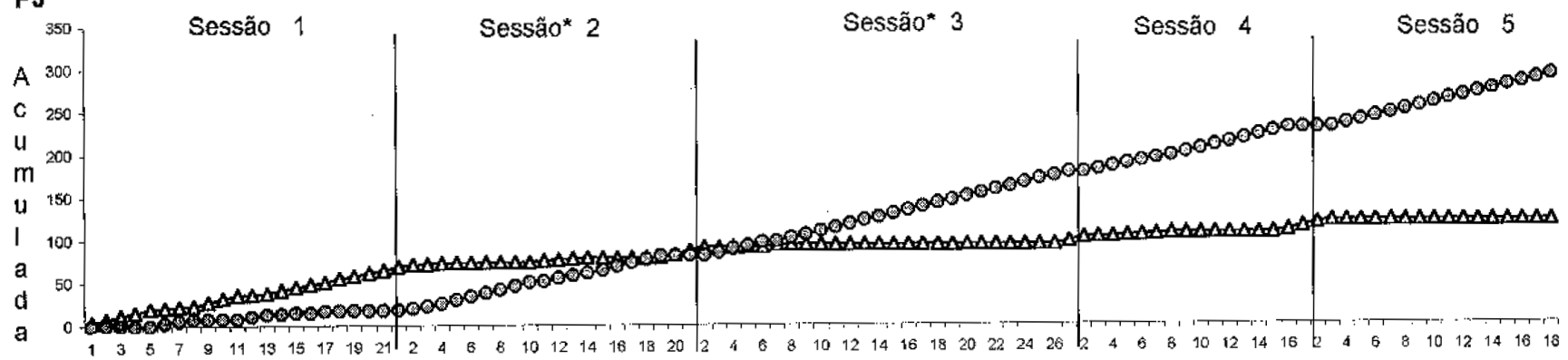

Figura 1. Freqüência das categorias de comportamento da dentista e de P3 durante as cinco primeiras sessões de atendimento. As categorias do dentista foram: Realiza Atividade, Direção, Tranqüilização, Distração, Persuasão e Punição Verbal. Em relação a P3 as categorias foram: Colaboração e Não Colaboração. Nas sessões com asteríscos a criança tinha recebido 0,3mg de Diazepam por $\mathrm{Kg}$ de peso.

\section{Legendas da Figura 1}
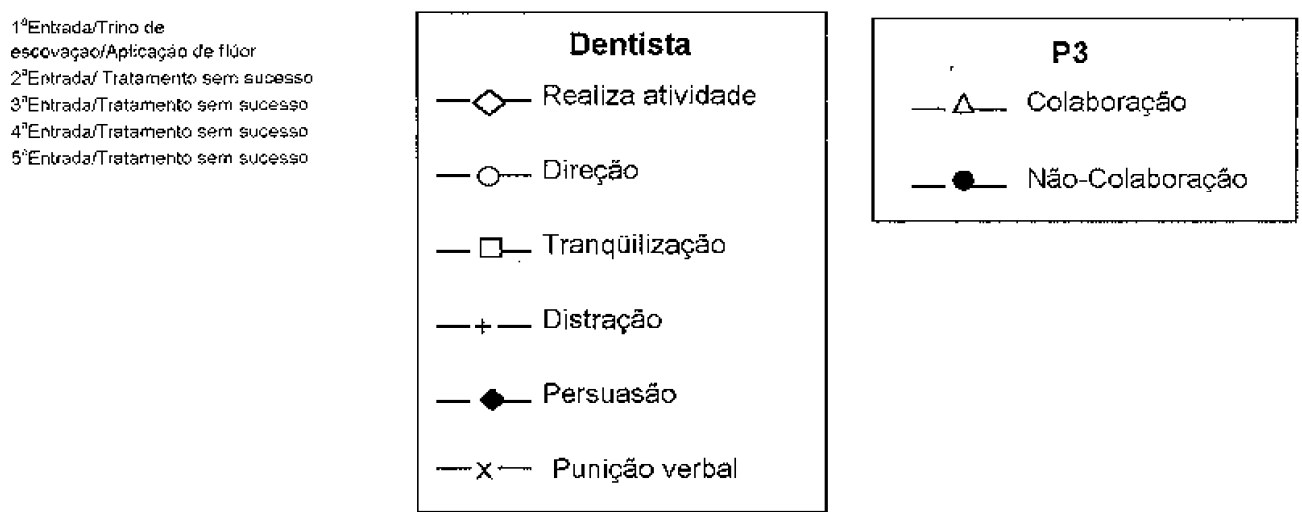


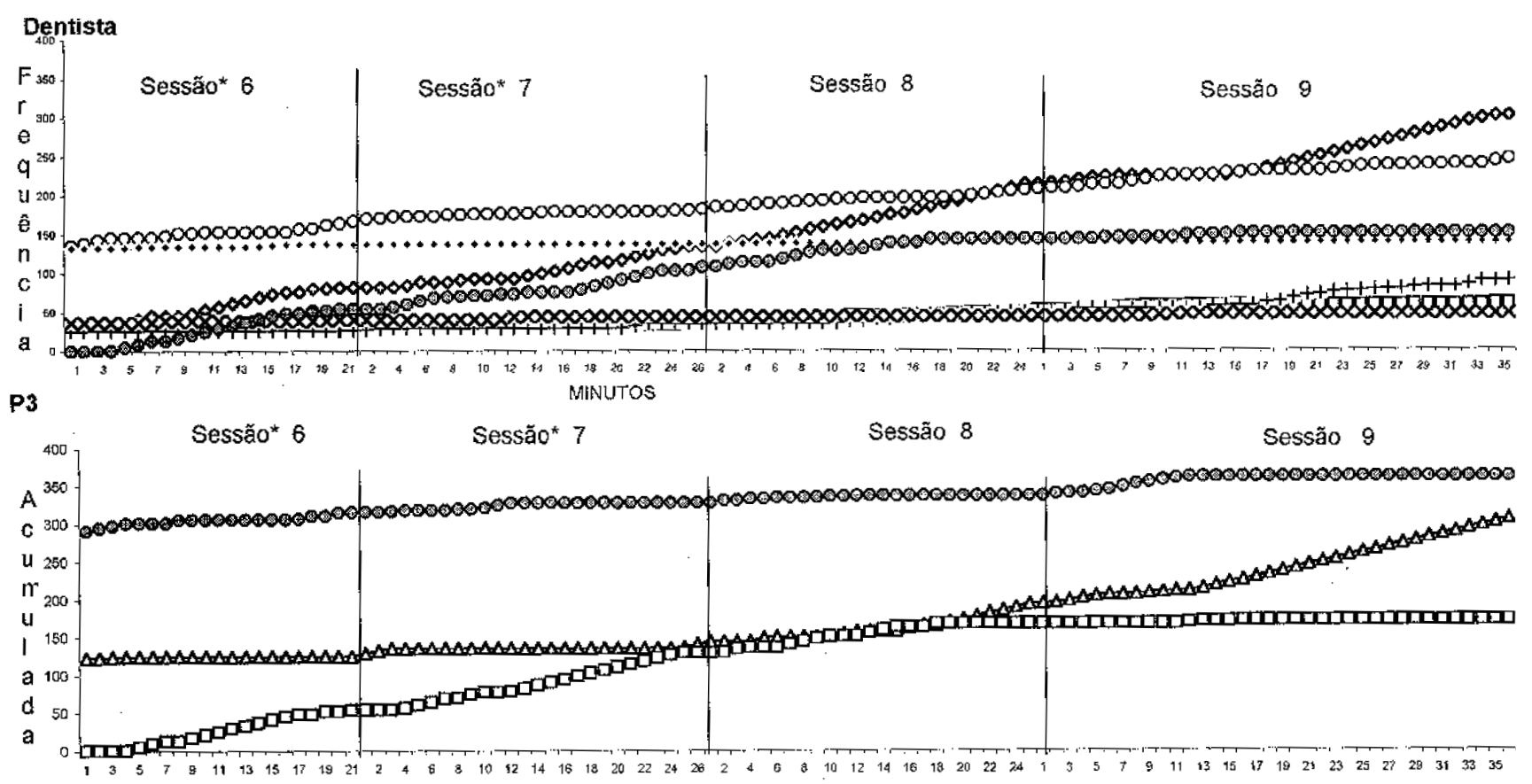

Minutos

Figura 2. Freqüência acumulada das categorias de comportamento da dentista e do P3 da sexta e nona sessões de atendimento. As categorias do dentista foram: Realiza Atividade, Direção, Tranqüilização, Distração, Persuasão, Punição Verbal e Restrição Física para o tratamento odontológico. Para o P3 as categorias foram: Colaboração, Colaboração sob Restrição Física e Não Colaboração. Nas sessões com asteríscos a criança tinha recebido 0,3mg de Diazepam por Kg de peso.

\section{Legendas da Figura 2}
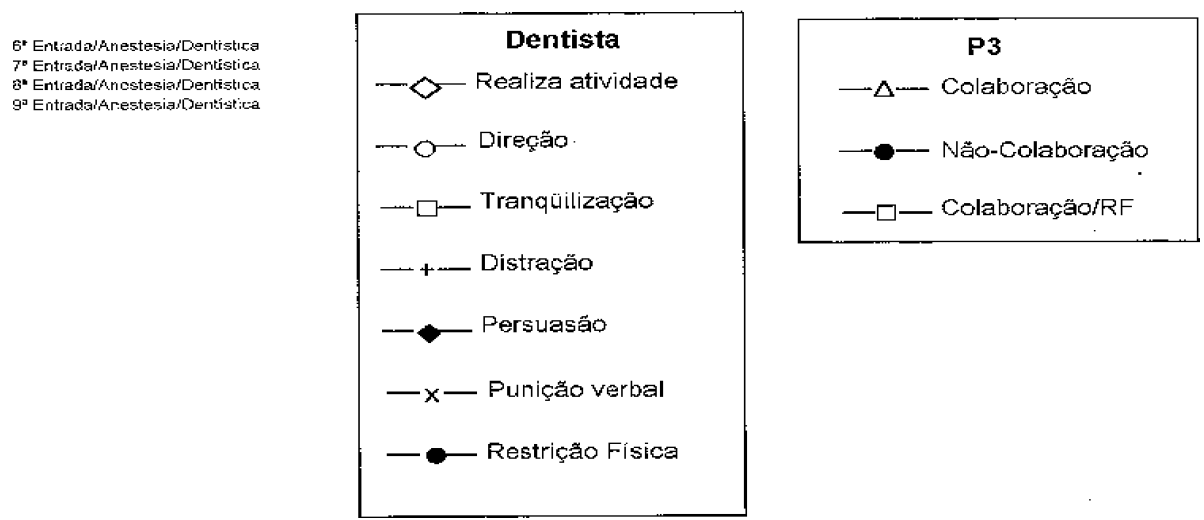

comportamental não se manteve. Observou-se um aumento da freqüência acumulada da categoria "colaboração sem restrição física" na oitava e nona sessões.

\section{Discussão}

Durante as cinco sessões de atendimento de P1, a profissional executou grande número de atividades clínicas (em 67,4\% do tempo) e, concomitantemente, utilizou estratégias positivas de manejo do comportamento (direção e distração) em 66,4\% do tempo. No decorrer de todo o atendimento odontológico de P1, a categoria direção foi a mais freqüente $(48,2 \%)$. Os comportamentos não concorrentes ocorreram em $92,7 \%$ do tempo, sugerindo um efeito benéfico da adoção de estratégias positivas pela dentista. As categorias de comportamento da dentista, em relação a P1, permitiram a obtenção da colaboração do paciente e a conseqüente realização do tratamento odontológico.

Em relação a P2, a análise mostrou que a profissional utilizou estratégias positivas em $87,2 \%$ do tempo de atendimento. A profissional apresentou grande produtividade clínica, uma vez que, em $85,9 \%$ do tempo de tratamento, estava executando alguma rotina odontológica.

Tanto para P1 como para P2, verificou-se um equilibrio entre a utilização de estratégias positivas e a execução de atividade clínica. Estes resultados são consistentes com o 
relatado de Wurster, Weinstein e Cohen (1979), que revelaram uma maior probabilidade da categoria de comportamento “orientação/direção" ser seguida por colaboração. Weinstein e colaboradores (1982) observaram que, em geral, os comportamentos indicadores de medo (movimentar, chorar, gritar, protestar e lamuriar) são menos freqüentes quando o dentista dirige e reforça positivamente o comportamento da criança.

Já durante o atendimento de P3, o repertório de comportamentos da dentista nas cinco primeiras sessões, foi predominantemente de estratégias positivas, que se revelaram ineficazes para a obtenção de respostas de colaboração por parte do paciente. É importante destacar que, embora estivesse impedida de utilizar restrição física, a dentista apresentou punição verbal em $9 \%$ do tempo das sessões. A utilização desta modalidade de estratégia aversiva, no entanto, não reverteu a tendência comportamental da criança em não colaborar com a execução das atividades clínicas previstas para o tratamento. A criança apresentou comportamentos concorrentes em 71,7\% do tempo.

Curiosamente, P3 mantinha ampla interação verbal com a dentista, sugerindo que a criança obtinha sucesso em atrasar a execução das intervenções clínicas. A partir da sexta sessão, a adoção de restrição física criou condições que inibiram as respostas de fuga e esquiva da criança e induziram a evocação de respostas de colaboração. Considerando que a restrição física foi utilizada na sexta, sétima e início da oitava sessão, sugere-se que o aumento da freqüência de comportamento colaborativo, a partir da oitava sessão, está associado à percepção da criança de que não mantinha controle sobre a rotina odontológica, a qual seria executada com ou sem restrição física.

Por outro lado, é possível observar que durante a sexta e a sétima sessão, P3 apresentou poucos comportamentos colaborativos, que foram seguidos por estratégias positivas da dentista, fazendo com que a criança percebesse que dispunha de repertório para enfrentar a situação de tratamento odontológico, aumentando a probabilidade de colaborar sem o uso de restrição física. Deve-se lembrar, também, que, a partir da sexta sessão, a criança participou da atividade lúdica, o que, por sua vez, pode ter exercido a função de situação estabelecedora para a aquisição de comportamentos colaborativos.

É importante observar, no entanto, que a adoção repetitiva de restrição física nem sempre é eficiente em obter uma gradativa colaboração da criança exposta a tratamento de saúde. Crianças excessivamente não colaboradoras e submetidas a contingências pouco efetivas à sensibilidade comportamental das mesmas podem ser expostas, desnecessariamente, à muito mais tempo de estimulação aversiva, proporcionando um aumento potencial da percepção de sofrimento e da freqüência de comportamentos concorrentes, conforme descrevem Christiano e Russ (1998).
A medicação ansiolítica utilizada não demonstrou efeitos sobre o repertório de comportamento dos pacientes. A comparação das sessões de linha de base com as sessões em que os pacientes receberam medicação ou placebo, não permitiu qualquer diferenciação topográfica ou funcional. Estes resultados são semelhantes aos encontrados por Possobon (2000), que utilizou a mesma dose de diazepam $(0,3 \mathrm{mg} / \mathrm{kg}$ de peso) em um estudo que investigou a manipulação comportamental e farmacológica sobre as respostas de não colaboração de seis pacientes odontológicos.

Caldana e Biasoli-Alves (1990) sugerem a necessidade do odontopediatra compreender o paciente em um contexto de desenvolvimento, reconhecendo que, durante a infância, a criança está mais vulnerável e sensível a seu ambiente. É essencial que o odontopediatra mantenha sempre presente perguntas sobre como e quando agir com a criança no consultório, de forma a adotar estratégias que facilitem a realização do tratamento odontológico. Moraes e Gil (1991) ressaltam que, existem circunstâncias em que a rebeldia da criança exige atitudes invasivas do profissional. A Academia Americana de Odontopediatria (1996) reconhece esta situação quando recomenda aos profissionais da área o uso de estratégias de "controle pela voz", "mão sobre a boca" e "restrição física" em circunstâncias em que o tratamento é absolutamente necessário e outras estratégias positivas já foram utilizadas, como foi o caso específico de P3. Vale a pena ressaltar que as recomendações da Academia Americana de Odontopediatria incluem outras estratégias como reforçamento, distração e conte-mostre-faça, que também foram adotadas no presente estudo e produziram comportamentos colaboradores em P1 e P2.

A pesquisa comportamental em situação odontológica, tem se baseado na suposição tácita de que a criança é o problema e seu comportamento deve ser alterado em beneficio da realização do tratamento (Moraes, 1999). Esta pressuposição tem levado ao desenvolvimento de trabalhos destinados a verificar a eficácia de estratégias para modificar o comportamento da criança, tais como distração (Stark \& colaboradores, 1989), distração contingente (Ingersoll, Nash \& Gramber, 1984), modelação por filme ou "ao vivo" (Melamed, Hawes, Heiby \& Glick, 1975; Stokes \& Kennedy, 1980), reforçamento de comportamentos adequados (Allen, Stark, Rigney, Nash \& Stokes 1988; Allen \& Stokes, 1987) e fuga contingente (Allen, Loiben, Allen \& Stanley, 1992). Em termos gerais, estas estratégias resultam em melhora na colaboração da criança, mas observa-se uma grande variabilidade nos valores da freqüência relativa de comportamento não-colaboradores nas sessões de linha de base e nas sessões de intervenção. Os autores atribuem pouca importância à uma análise que procure explicar essa variabilidade em termos de outras variáveis presentes na situação. Nesse sentido, o tratamento odontológico não precisa ser, necessariamente, sempre sentido como aversivo, 
sendo fundamental a identificação das variáveis comportamentais e odontológicas e sua interação ao longo de sessões sucessivas de tratamento. A aversividade de um procedimento ou de um padrão comportamental do dentista pode se alterar intra-sessão e entre sessões de atendimento. No entanto, a pesquisa na área não tem se preocupado em realizar análises de mudanças comportamentais que ocorrem ao longo do tempo e tem revelado uma ênfase tecnicista de busca de resultados o que, de certa maneira, empobrece a produção científica na área de odontologia comportamental.

Este trabalho representa uma contribuição da análise funcional do comportamento ao estudo da interação profissional - paciente em odontopediatria. Neste contexto, é importante salientar o conceito de relação comportamento - ambiente, por envolver a idéia básica de que o fundamental não é o desempenho, mas a relação estabelecida entre o desempenho comportamental e os eventos da situação investigada (Souza, 1997).

Os resultados obtidos permitem afirmar que a) é possível, por meio de uma análise funcional, identificar diferentes classes de comportamentos e variáveis controladoras dos comportamentos envolvidos na interação odontopediatra criança; e b) as condições de saúde bucal, o plano de tratamento, o nível de medo e o grau de colaboração das crianças podem ser considerados como condições que estabelecem comportamentos profissionais padronizados.

\section{Referências}

Academia Americana de Odontopediatria (1996). Guidelines for behavior management. Pediatric Dentistry (Special issue) 18, 86-88.

Allen, K. D., Loiben, T., Allen, S. J. \& Stanley, R. T. (1992). Dentist: Implemented contingent escape for management of disruptive child behavior. Journal of Applied Behavior Analysis, 25, 629-636.

Allen, K. D., Stanley, R. \& McPherson, K. (1990). Evaluation of behavior management technology dissemination in pediatric dentistry. Pediatric Dentistry, 12, 79-82.

Allen, K. D., Stark, L. J, Rigney, B. A., Nash, D. A. \& Stokes, T. F. (1988). Reinforced practice of children's cooperative behavior during restorative dental treatment. Journal of Dentistry for Children, 55, 273-277.

Allen, K. D. \& Stokes, T. F. (1987). Use of escape and reward in the management of young children during dental treatment. Journal of Applied Behavior Analysis, 20, 381-390.

Andrade, E. D. (1999). Terapêutica medicamentosa em odontologia. São Paulo: Artes Médicas.

Caldana, R. L. \& Biasoli-Alves, Z. M. (1990). Psicologia do desenvolvimento: Contribuição à odontopediatria. Revista de Odontologia da USP, 4, 256-260.
Christiano, B. \& Russ, S. W. (1998). Matching preparatory intervention to coping style: The effects on children's distress in the dental setting. Journal of Pediatric Psychology, 23, $17-27$.

Costa, Jr. A. L. (2001). Análise de comportamentos de crianças expostas à punção venosa para quimioterapia. Tese de Doutorado não-publicada, Programa de Pós-Graduação em Psicologia, Instituto de Psicologia, Universidade de Brasília. Brasília, DF.

Federation Dentaire Internationale/ Technical Report $n^{\circ} 32$ (1989). Premedication in Dentistry. International Dentistry Journal, 39, 55-61.

Fuchs, F. D. \& Wannamacher, L. (1992). Farmacologia clínica: Fundamentos para terapêutica racional. Rio de Janeiro: Guanabara Koogan.

Ingersoll, B. D., Nash, D. A. \& Gramber, C. (1984). The use of contingent audiotaped material with pediatric dental patients. Journal American Dental Association, 109, 717-720.

Kazdin, A. E. (1993). Evaluation in clinical practice: Clinically sensitive and systematic methods of treatment delivery. Behavior Therapy, 24, 11-45.

Melamed, B. G., Hawes, R. R., Heiby, E. \& Glick J. (1975). Use of filmed modeling to reduce uncooperative behavior of children during dental treatment. Journal of Dentistry Research, 54, 797-801.

Meyer, S. B. (1997). O conceito de análise funcional. Em M. Delitti (Org.), Sobre comportamento e cognição (pp. 31-36). São Paulo: ARBYtes.

Mindus, P. (1987). Anxiety, pain and sedation: Some psychiatry aspects. Acta Anaesthesiology Scandinavian Supplementum, 88, 7-12.

Moraes, A. B. A. (1999). Comportamento e saúde bucal: Circunscrevendo o campo. Em R. R. Kerbauy (Org.), Comportamento e saúde: Explorando alternativas (pp. 6183). Santo André, SP: Arbytes.

Moraes, A. B. A. \& Gil, I. (1991). Odontopediatria clínica. São Paulo: Santos.

Possobon, R. F. (2000). Uso combinado de estratégias comportamentais e farmacológicas no manejo da criança não-colaboradora durante o atendimento odontológico. Dissertação de Mestrado não-publicada, Curso de Pós-graduação em Odontologia: Área de Farmacologia, Anestesiologia e Terapêutica, Faculdade de Odontologia de Piracicaba, Universidade de Campinas. Piracicaba, São Paulo.

Prins, P., Veerkamp, J., Horst, G., Jong, A. \& Tan, L. (1987). Behavior of dentists and child patients during treatment. Community Dentistry of Oral Epidemiology, 15, 253257.

Souza, D. G. (1997). A evolução do conceito contingência? Em R. A. Banaco (Org.), Sobre comportamento e cognição (pp. 82-87). Santo André: Arbytes.

Stark, L. J., Allen, K. D., Hurst, M., Nash, D. A., Rigney, B. \& Stokes, T. F. (1989). Distraction: Its utilization and efficacy with children undergoing dental treatment. Journal of Applied Behavior Analysis, 22, 297-307.

Stokes, T. F. \& Kennedy, S. H. (1980). Reducing child uncooperative behavior during dental treatment through modeling and reinforcement. Journal of Applied Behavior Analysis, 13, 41-49.

Ten Berge, M., Veerkamp, J. \& Hoogstraten, J. (1999). Dentists' behavior in response to child dental fear. Journal of Dentistry for Children, 66, 36-40.

Trapp, L. D. (1991). Pharmacological management of pain and anxiety. Em E. A. Neidle \& J. A. Yagiela (Orgs.), Farmacologia e terapêutica para dentistas (pp.156-166, $3^{a}$ ed.). Rio de Janeiro: Guanabara Koogan.

Weinstein. P., Getz, T., Ratener, P. \& Domoto, P. (1982). The effect of dentist's behaviors on fear-related behaviors in children. Journal of American Dentistry Association, 104, 32-38.

Wurster, C., Weinstein, P. \& Cohen, A. (1979). Communication patterns in pedodontics. Perceptual and Motor Skills, 48, 150-166.

Recebido: 26/11/2002 $1^{a}$ Revisão: $12 / 05 / 2003$

Última Revisão: 01/07/2003

Aceite Final: 04/07/2003

Sobre os autores

Antonio Bento Alves de Moraes é Professor Titular de Psicologia Aplicada da Faculdade de Odontologia de Piracicaba da Universidade de Campinas. É Coordenador do Centro de Pesquisa e Atendimento Odontológico para Pacientes Especiais da mesma instituição.

Kira Anayansi Singh Sanchez é Doutora em Odontopediatria pela Universidade Júlio Mesquita Filho da Universidade Estadual Paulista.

Rosana de Fátima Possobon é Coordenadora Clínica do Centro de Pesquisa e Atendimento Odontológico para Pacientes Especiais (Cepae) da Faculdade de Odontologia de Piracicaba da Universidade de Campinas. É Doutora em Odontologia pela mesma instituição.

Áderson Luiz Costa Júnior é Professor Adjunto do Instituto de Psicologia da Universidade de Brasília. 